\title{
Macrophage plasticity and polarization: in vivo veritas
}

\author{
Antonio Sica ${ }^{1,2}$ and Alberto Mantovani ${ }^{1,3}$ \\ ${ }^{1}$ Istituto Clinico Humanitas IRCCS, Rozzano, Italy. ${ }^{2}$ DiSCAFF, University of Piemonte Orientale A. Avogadro, Novara, Italy. \\ ${ }^{3}$ Department of Translational Medicine, University of Milan, Milan, Italy.
}

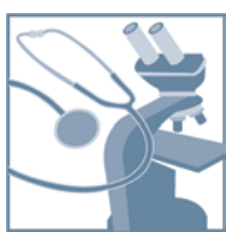

Diversity and plasticity are hallmarks of cells of the monocyte-macrophage lineage. In response to IFNs, Toll-like receptor engagement, or IL-4/IL-13 signaling, macrophages undergo M1 (classical) or M2 (alternative) activation, which represent extremes of a continuum in a universe of activation states. Progress has now been made in defining the signaling pathways, transcriptional networks, and epigenetic mechanisms underlying M1-M2 or M2-like polarized activation. Functional skewing of mononuclear phagocytes occurs in vivo under physiological conditions (e.g., ontogenesis and pregnancy) and in pathology (allergic and chronic inflammation, tissue repair, infection, and cancer). However, in selected preclinical and clinical conditions, coexistence of cells in different activation states and unique or mixed phenotypes have been observed, a reflection of dynamic changes and complex tissue-derived signals. The identification of mechanisms and molecules associated with macrophage plasticity and polarized activation provides a basis for macrophage-centered diagnostic and therapeutic strategies.

\section{Introduction}

Macrophages are an essential component of innate immunity and play a central role in inflammation and host defense (1). Moreover, these cells fulfill homeostatic functions beyond defense, including tissue remodeling in ontogenesis and orchestration of metabolic functions (1-3).

Cells of the monocyte-macrophage lineage are characterized by considerable diversity and plasticity. In tissues, mononuclear phagocytes respond to environmental cues (e.g., microbial products, damaged cells, activated lymphocytes) with the acquisition of distinct functional phenotypes. In response to various signals, macrophages may undergo classical M1 activation (stimulated by TLR ligands and IFN- $\gamma$ ) or alternative M2 activation (stimulated by IL-4/IL-13); these states mirror the Th1-Th2 polarization of T cells $(2,4)$.

The M1 phenotype is characterized by the expression of high levels of proinflammatory cytokines, high production of reactive nitrogen and oxygen intermediates, promotion of Th1 response, and strong microbicidal and tumoricidal activity. In contrast, M2 macrophages are considered to be involved in parasite containment and promotion of tissue remodeling and tumor progression and to have immunoregulatory functions. They are characterized by efficient phagocytic activity, high expression of scavenging molecules, the expression of mannose and galactose receptors, production of ornithine and polyamines through the arginase pathway, and an IL-12 ${ }^{\text {lo } I L-10 ~}{ }^{\text {hi }}$ IL-1decoyR ${ }^{\text {hi IL-1RA }}{ }^{\text {hi }}$ phenotype $(1,4)$. M1-M2 macrophages also are distinct in their chemokine expression profiles.

Signals including IL-10, glucocorticoid hormones, molecules released from apoptotic cells, and immune complexes also profoundly affect monocyte-macrophage function. These signals induce expression of functional phenotypes that share selected properties with M2 cells (e.g., high mannose and scavenger receptor expression), but are distinct from them, for instance, in terms of the chemokine repertoire. Operationally, we refer to these cells as M2 like (5).

Conflict of interest: Antonio Sica has received grant support from Johnson \& Johnson.

Citation for this article: J Clin Invest. 2012;122(3):787-795. doi:10.1172/JCI59643.
Plasticity and flexibility are key features of mononuclear phagocytes and of their activation states $(2,4,6)$. The phenotype of polarized M1-M2 macrophages can, to some extent, be reversed in vitro and in vivo $(7,8)$. Moreover, pathology is frequently associated with dynamic changes in macrophage activation, with classically activated M1 cells implicated in initiating and sustaining inflammation and M2 or M2-like cells associated with resolution or smoldering chronic inflammation (9). It remains unclear whether the mechanism of these switches involves the recruitment of circulating precursors or the reeducation of cells in situ. However, it is now apparent that specialized or polarized T cells (Th1, Th2, Tregs) that are key orchestrators of macrophage polarized activation (2) also exhibit previously unsuspected flexibility and plasticity (10).

Here, we will focus on recent progress in understanding the molecular basis underlying macrophage polarization, including signaling pathways, transcription factors, and epigenetic regulation. Moreover, the dynamics and limitations in our understanding of polarized macrophage activation in vivo will be discussed, focusing on selected pathological conditions (for references to pathology not discussed here, see Supplemental References; supplemental material available online with this article; doi:10.1172/ JCI59643DS1). Previous reviews also provide a framework for this work $(1-3,6,11)$.

\section{Molecular determinants of macrophage polarization}

A network of signaling molecules, transcription factors, epigenetic mechanisms, and posttranscriptional regulators underlies the different forms of macrophage activation. Canonical IRF/STAT signaling pathways are activated by IFNs and TLR signaling to skew macrophage function toward the M1 phenotype (via STAT1) or by IL-4 and IL-13 to skew toward the M2 phenotype (via STAT6) (3). M1 macrophages upregulate IRF5, which is essential for induction of cytokines (IL-12, IL-23, TNF) involved in eliciting Th1 and Th17 responses (12). The IL-4 type I and type II receptors $(1,13)$ activate Stat6, which in turn activates transcription of genes typical of M2 polarization, e.g., mannose receptor (Mrc1), resistin-like $\alpha$ 


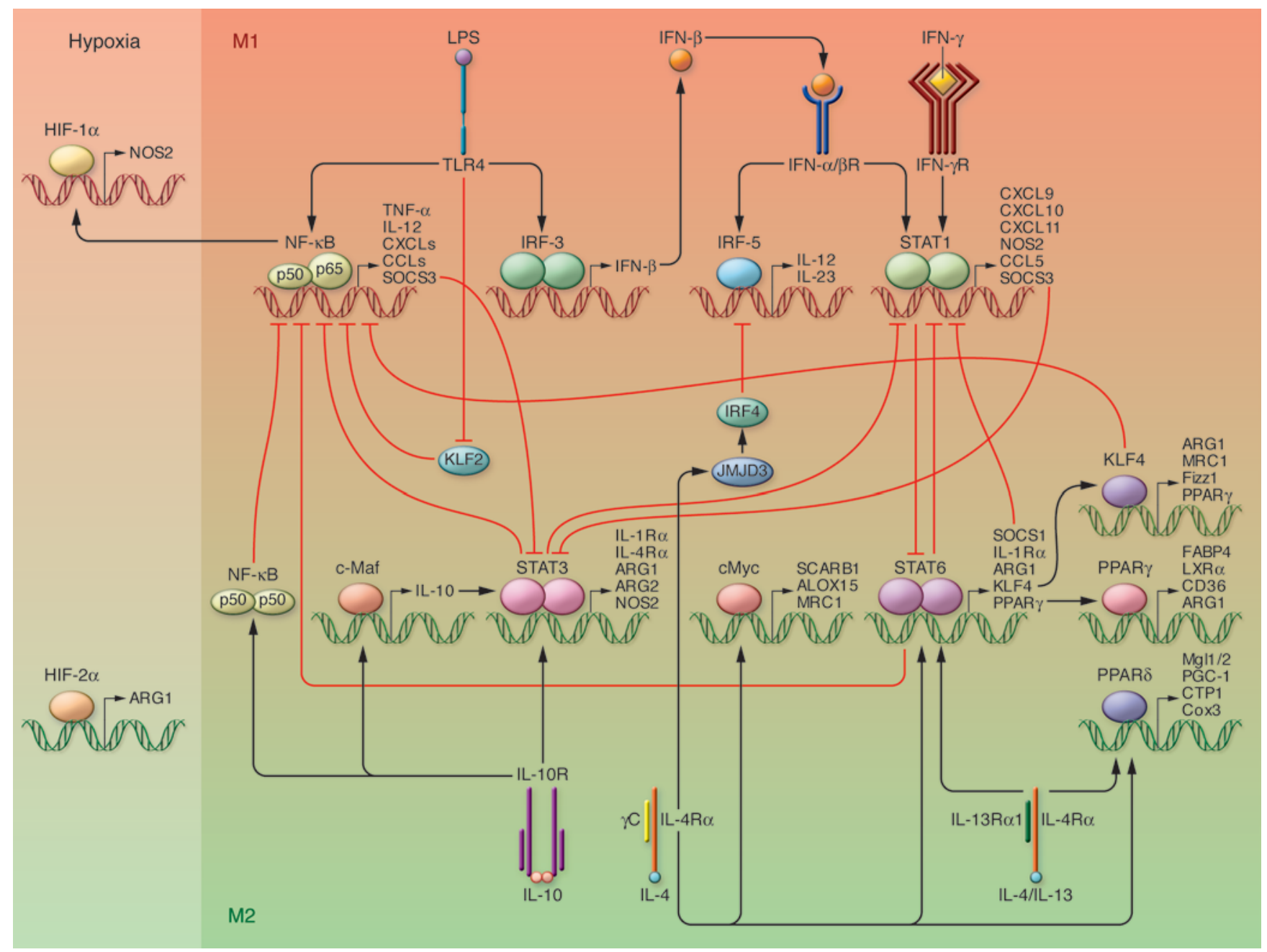

\section{Figure 1}

Mechanisms of macrophage polarization. The major pathways of macrophage polarization are outlined. The crosstalk between the M1-M2 macrophage-polarizing pathways is also indicated. The balance between activation of STAT1 and STAT3/STAT6 finely regulates macrophage polarization and activity. A predominance of NF- $\mathrm{kB}$ and STAT1 activation promotes M1 macrophage polarization, resulting in cytotoxic and inflammatory functions. In contrast, a predominance of STAT3 and STAT6 activation results in M2 macrophage polarization, associated with immune suppression and tumor progression. PPAR $\gamma$ and PPAR $\delta$ control distinct aspects of M2 macrophage activation and oxidative metabolism. KLF4 and KLF2 participate in the promotion of M2 macrophage functions by cooperating with STAT6 and suppressing the NF-KB/HIF- $1 \alpha-$ dependent transcription, respectively. IL-4-induced c-Myc activity controls a subset of M2-associated genes. IL-4 also induces the M2-polarizing Jmjd3-IRF4 axis to inhibit IRF5-mediated M1 polarization. IL-10 promotes M2 polarization through the induction of p50 NF-KB homodimer, c-Maf, and STAT3 activities.

(Retnla, Fizz1), and chitinase 3-like 3 (Chi3l3, Ym1) (14). IL-10 activates STAT3-mediated expression of genes (Il10, Tgfb1, Mrc1) associated with an M2-like phenotype $(4,15,16)$.

STAT-mediated activation of macrophages is regulated by members of the SOCS family. IL-4 and IFN- $\gamma$, the latter in concert with TLR stimulation, upregulate SOCS1 and SOCS3, which in turn inhibit the action of STAT1 and STAT3, respectively $(17,18)$.

Downstream of, or in parallel with, the IRF/STAT/SOCS pathway, a panel of transcription factors orchestrates polarized macrophage activation. The nuclear receptors PPAR $\gamma(19)$ and PPAR $\delta(20,21)$ control distinct subsets of genes associated with M2 macrophage activation and oxidative metabolism (Figure 1). Interestingly, STAT6 coordinates and synergizes with both PPAR $\gamma(22)$ and Krüppel-like factor 4 (KLF4), a member of a family of proteins that contribute to macrophage function $(23,24)$. KLF4 cooperates with Stat 6 to induce M2 genes (Arg-1, Mrc1, Fizz1, PPAR $\gamma$ ) and inhibit M1 genes (TNFa, Cox-2, $C C L 5, i N O S)$ via sequestration of coactivators required for NF- $\mathrm{KB}$ activation. KLF2 regulates macrophage activation by inhibiting NF-КB/ HIF-1 $\alpha$ activities (25). IL-4 also induces c-Myc activity in human macrophages (26), which controls genes of M2 activation (Scarb1, Alox15, and Mrc1) as well as STAT6 and PPAR $\gamma$ activation (26).

TLR engagement leads to NF- $\kappa \mathrm{B}$ activation and production of inflammatory mediators (27) associated with M1 macrophages. However, NF-кB activation also activates a genetic program essential for resolution of inflammation (28) and for M2 polarization of tumor-associated macrophages (TAMs) (29). Moreover, induction of p50 NF- $\kappa \mathrm{B}$ homodimers is essential for M2 polarization in vitro and in vivo (30). The hypoxia-inducible factors HIF- $1 \alpha$ and HIF-2 $\alpha$ are expressed differentially in M1- and M2-polarized macrophages (31) and regulate inducible NOS2 (M1) and arginase 1 (M2), respectively. 
Epigenetic changes and noncoding RNAs also participate in directing macrophage polarization (32-34). IL-4 induces upregulation of the histone demethylase JMJD3 in mouse macrophages, which alters chromatin modifications to promote expression of M2 genes and inhibit M1 genes. miR-155 was recently identified as targeting the IL-13R $\alpha 1$ subunit, thus decreasing a set of M2 genes in human macrophages (35).

\section{Pathology}

Resolution of inflammation and repair. Remodeling and repair occur dynamically during ontogenesis and inflammation, and these processes are orchestrated by macrophages.

Macrophages undergo dynamic changes during different phases of wound healing. M1-polarized macrophages mediate tissue damage and initiate inflammatory responses $(1,2)$. During the early stages of the repair response after wounding the skin, infiltrating macrophages have an M2 phenotype and their depletion inhibits the formation of a highly vascularized, cellular granulation tissue and of scar tissues (36). Under these conditions, the removal of apoptotic cells (efferocytosis) $(37,38)$ and the presence of TGF- $\beta$ (39) may skew macrophage function, though demonstration of actual in vivo relevance of these findings is lacking.

In a peritoneal model of inflammation, resolution phase macrophages expressed a unique mixed M1-M2 phenotype, and cAMP was essential to restrain M1 activation (40). In humans, chronic venous ulcers (CVU) represent a failure to resolve a chronic inflammatory condition (41). Correlative analysis in patients and a mouse model suggested that in CVU the infiltrating macrophages fail to switch from an M1 to an M2 phenotype (41). Notably, iron metabolism is differentially regulated in polarized macrophages (42). In CVU, the iron overload in macrophages sustained their M1 activation, leading to ROS-mediated DNA damage, fibroblast cellular senescence, and defective tissue repair (41). It is tempting to speculate that similar mechanisms may underlie M2 functions in patients with severe burns, where hemorrhage and tissue damage, along with the high release of iron by M2 macrophages (42), may result in high iron tissue levels (43).

Dynamic changes in the phenotype of recruited mononuclear phagocytes have been observed in models of ischemic heart disease (44), suggesting that this is a general feature of the natural history of repair processes. In a murine model of hind limb ischemia, haplodeficiency of the oxygen sensor prolyl-hydroxylase PHD2 induced the canonical NF-KB pathway in macrophages, which promoted their M2 polarization and proarteriogenic phenotype (45). This observation may suggest a functional link between oxygen deprivation, as occurring in wounds and tumors, and progressive induction of proarteriogenic M2 macrophages. Thus, although the macrophage phenotype in resolution need not be a phenocopy of in vitro-generated M2 cells, preclinical and clinical evidence support the long-held view of a key role of polarized macrophages in tissue repair.

In a model of retinal neuropathy, mononuclear phagocyte infiltration generates a neuroprotective microenvironment promoting retinal progenitor cell survival (46). The interplay of polarized macrophages with stem and progenitor cells is likely a key component of their role in repair and remodeling, although the actual tissue-protective significance of polarized macrophages in degenerative diseases and their involvement with stem and progenitor cells remain to be determined (see Supplemental References).

Macrophage activation has been found in autoimmune and inflammatory diseases and in particular in lupus nephritis
(47). In a murine model of SLE, proinflammatory activation of macrophages was sustained by Notch signaling (48). Conversely, the acute phase protein serum amyloid P (SAP) skewed macrophages toward an antiinflammatory M2-like phenotype, thus alleviating lupus nephritis (49). In RA, a major source of M1 cytokines (TNF- $\alpha$, IL-1 $\beta$, IL-12p70) (50) in the joints are the synovial macrophages, whose number correlate with the inflammatory disease activity (51). On this basis, PPAR $\delta$ and PPAR $\gamma$ are considered potential disease-modifying drugs for RA $(52,53)$. In a murine model of experimental colitis, recruitment of CCL11-expressing Ly6C ${ }^{\text {hi }}$ CCR2 ${ }^{+}$inflammatory monocytes into the colon correlates with eosinophil infiltration and histopathology (54).

\section{Infection}

Bacteria. M1 macrophages are generally considered responsible for resistance against intracellular pathogens and characterize infection with Listeria monocytogenes (55), Salmonella typhi, and Salmonella typhimurium (56), as well as the early phases of infection with Mycobacterium tuberculosis (57), Mycobacterium ulcerans, and Mycobacterium avium $(58,59)$. Uncontrolled M1 inflammation associated with acute infections with E. coli or Streptococcus sp. causes gastroenteritis, urinary tract infections, neonatal meningitis, and sepsis (60). The M1-M2 switch observed during the transition from acute to chronic infection may provide protection against overwhelming uncontrolled inflammation; however, a phenotype switch can also favor pathogens that have evolved strategies to interfere with M1-associated killing (58, 61-64).

Chronic inflammation and granuloma formation are characterized by the presence of macrophages with an epithelial morphology and by macrophage homotypic fusion with the formation of multinucleated giant cells (MNG) (65). IL-4-mediated upregulation of E-cadherin, which engages in intercellular homotypic adhesion, is likely to underlie MNG formation. STAT6 drives expression of E-cadherin and DC transmembrane protein (DC-STAMP; ref. 66) during MNG generation. The actual function and role of MNG remain elusive.

Parasites. In experimental and human parasite infections, macrophages generally undergo a dynamic switch toward M2 polarization $(61,67)$. The early and late phases of Taenia crassiceps infection are characterized by Th1-driven M1 and Th2-driven IL-4-mediated M2 polarization of macrophages, respectively $(11,68)$. A similar M1-M2 switch has been reported during Schistosoma mansoni and Trypanosoma congolense infection (69). In the chronic stage of T. crassiceps infection, the $\mathrm{p} 50 \mathrm{NF}-\mathrm{\kappa B}$ subunit (see above) promotes protective $\mathrm{M} 2$ macrophage polarization associated with decreased parasite burden (30).

The recurrent association of M2 polarization with parasite infections does not necessarily imply a generalized relevance in pathogenesis. For instance, lineage-selective ablation of the IL- $4 \alpha$ chain causes a dramatic increase in susceptibility to S. mansoni, but has no discernible effect on Nippostrongylus infection (70).

Viral infections. Polarized activation of macrophages has been associated with virus infection (e.g., HIV, Kaposi sarcoma-associated herpes virus, and others; see Supplemental References), and this polarization may be important in containing and limiting tissue damage. In particular, during severe respiratory syncytial virus-induced (RSV-induced) bronchiolitis, IL-4R $\alpha /$ STAT6dependent M2 macrophage differentiation reduces inflammation and epithelial damage in lungs (71). 
Table 1

Prototypic therapeutic strategies targeting macrophage polarization

$\begin{array}{lc}\begin{array}{l}\text { Function } \\ \text { Recruitment }\end{array} & \text { Therapeutic agent } \\ & \text { Anti-CSF-1 (Ab and antisense-ODN) } \\ \text { Anti-CCL2 (Ab) } & \text { CCL2 inhibitor (bindarit) } \\ \text { fms-tyrosine kinase inhibitors } & \text { Trabectedin } \\ \text { IFN- } \gamma & \text { TLR9 agonist (CpG-ODN) } \\ \text { Depletion } & \text { CpG-ODN + anti-IL-10 } \\ \text { Switch to M1 } & \text { Anti-CD40 (agonist) } \\ & \text { STAT3 inhibitors } \\ & \text { STAT6 inhibitors } \\ & \text { p50 NF- } \mathrm{KB} \text { inhibitors } \\ \text { IKK } \beta \text { inhibitors } \\ \text { Inhibition of M1 pathways } & \text { Zoledronic acid } \\ \text { Inhibition of M2 pathways } & \text { CD40 (antagonist) } \\ \text { Induction of M2 pathways } & \text { IL-33 (antagonist) } \\ & \text { PPAR } \gamma \text { agonists (thiazolidinediones) } \\ & \text { Nonthiazolidinedione tyrosine-based } \\ \text { PPAR } \gamma \text { ligand (GW7845) } & \text { Statin }\end{array}$

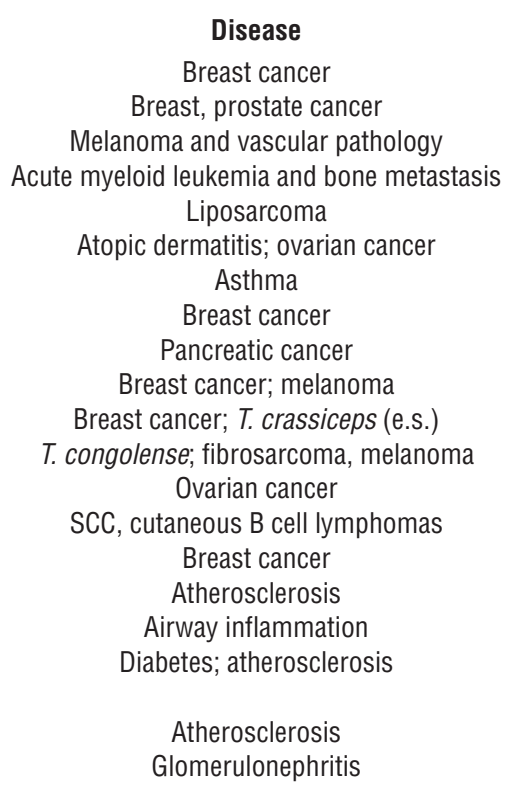

Breast cancer

Breast, prostate cancer

Melanoma and vascular pathology

Acute myeloid leukemia and bone metastasis Liposarcoma

Atopic dermatitis; ovarian cancer Asthma

Breast cancer

Pancreatic cancer

Breast cancer; melanoma

Breast cancer; T. crassiceps (e.s.)

T. congolense; fibrosarcoma, melanoma Ovarian cancer

SCC, cutaneous B cell lymphomas

Breast cancer

Atherosclerosis

Airway inflammation

Diabetes; atherosclerosis

Atherosclerosis

Glomerulonephritis

ODN, oligodeoxyribonucleotides; IKK $\beta$, IKK $\beta$ subunit of ІКB kinase (IKK); e.s., early stage of infection. For an extensive reference list, see Supplemental References.

\begin{abstract}
Allergy
Allergy is driven by Th2 cells and products and is associated with M2 polarization of macrophages $(72,73)$. IL-4-inducible chemokines acting on CCR4 (e.g., CCL22) have also been reported to promote skewing of macrophage function (74). Evidence now indicates that chitin- and arginase-dependent M2 pathways play an active role in pathogenesis $(75,76)$. Asthma is associated with tissue remodeling, including collagen deposition and goblet cell hyperplasia. IL-4-driven M2 polarization is likely to play a key role as an orchestrator of these processes (77).

Allergy represents a paradigm for IL-4/IL-13-driven type 2 inflammation. However, there is evidence that the inflammasome/IL-1/Th17 pathway can also drive allergic inflammation $(78,79)$. Moreover, a Th1-associated cytokine, IL-18, has also been implicated in allergic inflammation (80). It is therefore perhaps not surprising that mixed phenotype macrophages have been observed (81).
\end{abstract}

\section{Cancer}

Cancer-related inflammation is characterized by the recruitment of cells of the monocyte-macrophage lineage to tumor tissues $(2,82,83)$, which also condition the premetastatic niche, to favor secondary localization of cancer. Classically activated M1polarized macrophages have the potential to exhibit antitumor activity and to elicit tumor tissue disruption (4). At least in some models of carcinogenesis in the mouse, progression is associated with a phenotype switch from M1 to M2 (84). Th1-driven macrophage activation was found to mediate elimination of senescent hepatocytes, which drive subsequent carcinogenesis (85). It is therefore likely that classically activated M1 macrophages contribute to the $\mathrm{T}$ cell-mediated elimination and equilibrium phases during tumor progression (86).
At later stages of progression in mice and humans, TAMs generally have an M2-like phenotype with low IL-12 expression, high IL-10 expression, and low tumoricidal activity and promotion of tissue remodeling and angiogenesis. TAM infiltration is generally associated with poor prognosis, as shown in Hodgkin disease, glioma, cholangiocarcinoma, and breast carcinoma $(87,88)$. However, TAMs with various functional states can coexist in the same tumor $(2,89)$. Various pathways orchestrate the protumor function of myelomonocytic cells, including tumor-derived and host-derived signals (2). Lymphocytes are key orchestrators of TAM function (2), but pathways differ in tumors originating in different organs. For instance, skewing of macrophage function was found to be mediated by IL-4-producing Th2 cells in skin $(90,91)$ and by antibody-producing B cells in breast carcinogenesis $(92,93)$. B1 cells can promote cancer progression in the skin (5), and fibroblasts can also contribute to circuits driving macrophage polarization and tumor promotion (94).

Tumor cell products, including extracellular matrix components, IL-10, CSF-1, and chemokines (CCL2, CCL18, CCL17, and CXCL4), set macrophages in an M2-like, cancer-promoting mode $(82,95-97)$. TAMs can also interact with and promote the tumorigenicity of cancer stem cells (CSCs) via production of the milk-fat globule-epidermal growth factor-VIII (MFG-E8) by activation of the STAT3 and sonic hedgehog pathways (98).

The identification of the various cellular and molecular pathways that participate in inflammation in different human cancers will be required to translate our understanding of cancer-related inflammation to meaningful therapeutic advances.

\section{Obesity and metabolism}

Obesity-associated insulin resistance, diabetes, and metabolic syndrome are sustained by chronic subclinical inflammation (99). Adipose tissue macrophages (ATMs) are a major component 


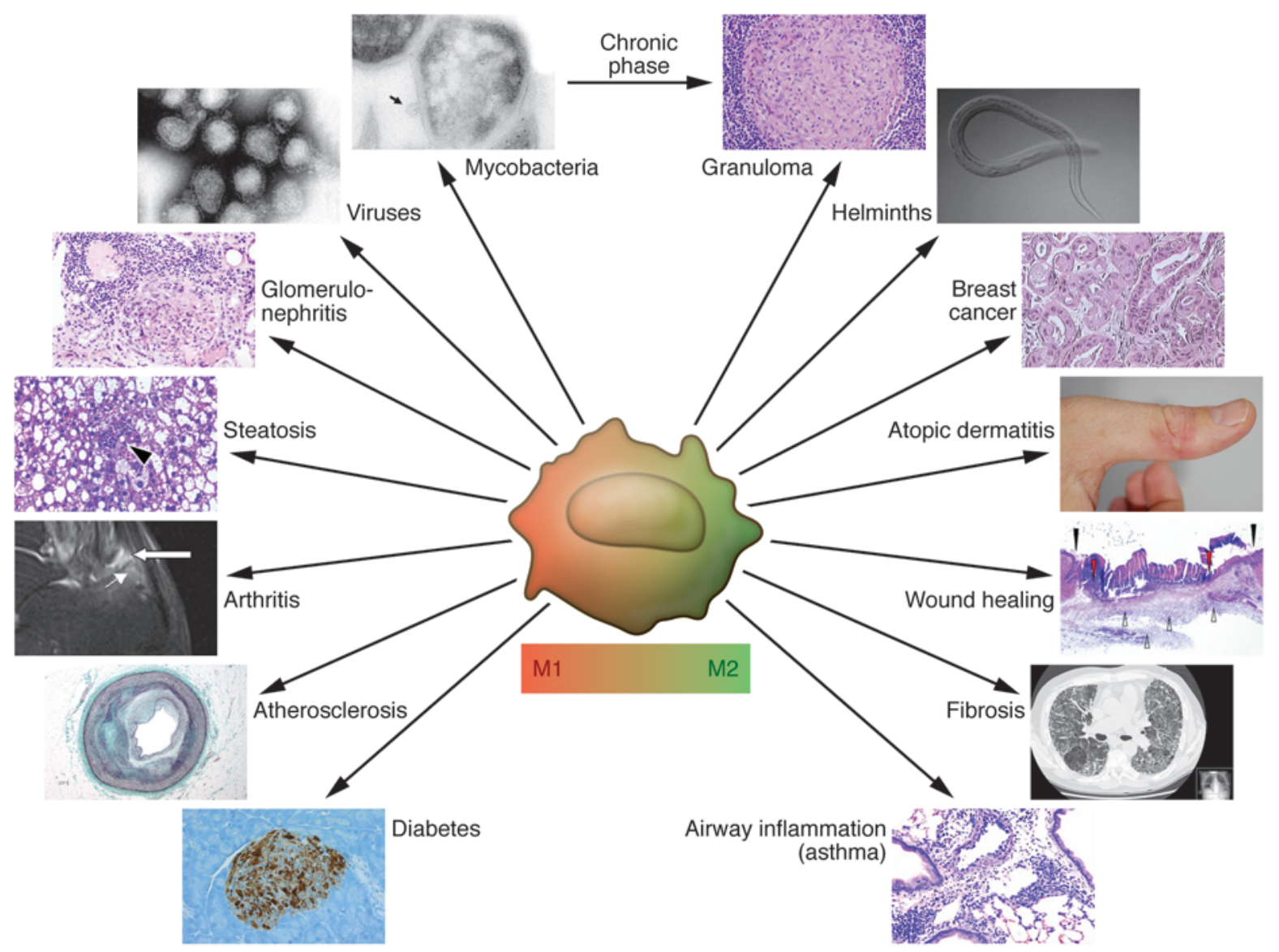

Figure 2

Schematic representation of macrophage plasticity and polarization in pathology. Dynamic changes occur over time with evolution of pathology: for instance, a switch from M1 to M2 macrophage polarization characterizes the transition from early to chronic phases of infection. Moreover, mixed phenotypes or populations with different phenotypes can coexist. For pathologies not discussed in the text, see Supplemental References. Images adapted from refs. 141-144.

of adipose tissue and are important players in obesity-associated pathology. In obese subjects and mice, adipocytes release mediators such as CCL2, TNF, or free fatty acids, which promote the recruitment and activation of ATMs (100-102). In turn, ATM-produced inflammatory cytokines (e.g., TNF, IL-6, IL-1 $\beta$ ) counteract the insulin-sensitizing action of adiponectin and leptin, leading to insulin resistance. ATM infiltration correlates with the degree of obesity (101-103). ATM accumulation is orchestrated by selected chemokines and their receptors (e.g., CCL2/CCR2 or CCL5/CCR5) $(101,103,104)$ and by the macrophage-derived apoptosis inhibitor of macrophages (AIM) (105).

ATMs from obese mice and humans are polarized toward an M1 phenotype, with upregulation of TNF and iNOS. In contrast "lean" ATMs express high levels of M2 genes, including IL-10, Ym1, and Arginase 1 (106, 107). Weight loss is associated with a shift back to an M2-like phenotype. However, evidence indicates that the ATM population in obese patients is diverse, with a minor $\mathrm{F} 4 / 80^{+} \mathrm{CD} 11 \mathrm{~b}^{+} \mathrm{CD} 11 \mathrm{c}^{-}$, IL-10 ${ }^{\text {hi }}$, M2-like population coexisting with M1 cells (106). Moreover, analysis of CD $11 c^{+}$ATMs from obese mice revealed a mixed profile of several M1-M2 gene transcripts (108). Weight loss is associated with an increase in $\mathrm{F} 4 / 80^{+} \mathrm{CD} 11 \mathrm{c}^{-} \mathrm{CD} 301^{+} \mathrm{M} 2$-like macrophages, which promote lipolysis (109).
Progress has been made in defining the molecular pathways that account for polarization of ATMs $(19,20,24,110,111)$ in obesity. Mice with macrophage-selective genetic inactivation of PPAR $\gamma$, PPARS, and KLF4 show inhibition of obesity-induced insulin resistance $(19,20,24,106,110)$, although these results may be strain specific (112). The adipose tissue of obese subjects shows a marked reduction in KLF4 levels. Nguyen et al. have recently demonstrated that adaptation to lower temperatures (thermogenesis) is associated with polarization of brown adipose tissue (BAT) and white adipose tissue (WAT) macrophages to the alternative state (M2), with increased expression of thermogenic genes (Ppargc1 and $U c p 1)$ (113). In response to cold, IL-4-driven M2 macrophages release noradrenaline in BAT and WAT, which coordinates fatty acid mobilization and energy expenditure (113). Although further work is needed to dissect the diversity and dynamics of ATMs, M2-like cells in nonobese individuals are likely involved in maintaining adipose tissue homeostasis, preventing inflammation, and promoting insulin sensitivity. In contrast, M1-like ATMs drive obesity-associated inflammation and insulin resistance (107). The role of macrophages in obesity and associated disorders underlines a homeostatic function of macrophages in metabolism as cells capable of reorienting their own metabolic activity and as orchestrators of general metabolism. 


\section{Therapeutic targets in polarized inflammation}

Specific macrophage-targeted therapies are now taking the first steps into the clinical arena (Table 1 ). In addition, therapeutic approaches not originally designed as macrophage oriented or specific have been found to affect macrophage activation and polarization. These off-target examples provide insights and lessons for the development of more specifically directed approaches.

Recruitment is a key determinant sustaining macrophage numbers at sites of inflammation and immunity. Monocyte attractants include members of the chemokine superfamily, CCL2/MCP-1 in particular, and growth factors interacting with tyrosine kinase receptors CSF-1 and VEGF $(83,114,115)$. CSF-1 receptor ( $\mathrm{c}-\mathrm{fms}$ ) kinase inhibitors have been generated, and these molecules exhibit antiangiogenic and antimetastatic activity in acute myeloid leukemia and melanoma models $(116,117)$. Chemokines and CSF-1 are more than monocyte attractants; they also promote M2-like skewing of macrophage function (97, $118,119)$. Antibodies directed against CCL2/CCR2 have proven active in prostate and breast cancer $(83,120)$. A CCL2 inhibitor (bindarit) has proven active in preclinical models of cancer and vascular pathology, resulting in inhibition of monocyte recruitment (121); this agent is now undergoing evaluation for clinical use. Anti-CSF-1 antibodies and antisense oligonucleotides suppress macrophage infiltration and xenograft mammary tumor growth in mice (122-124). VEGF inhibitors can decrease macrophage recruitment, and this effect may contribute to their antiangiogenic activity (125). In contrast, in response to neoadjuvant chemotherapy, altered composition in the immune microenvironment was found in breast cancer patients, with increased percentage of infiltrating myeloid cells (126).

Recent results suggest that in situ, proliferation is a key determinant of macrophage accumulation during inflammation in the peritoneum and lungs $(127,128)$. In the latter, IL-4 was found to sustain macrophage proliferation. If proliferation is indeed a major general mechanism sustaining macrophage accumulation, this may suggest novel approaches to reduce macrophage numbers in situ and a different perspective for anti-CSF-1/CSF-1R strategies. However, determining the actual significance of macrophage proliferation in humans, in particular in Th2-mediated inflammation, is a major stumbling block in this perspective.

Reorienting and reshaping deranged macrophage polarization is the holy grail of macrophage therapeutic targeting (129). Polarized phenotypes are reversible in vitro and in vivo (29, 130-132). In proof of concept and in a large clinical study in ovarian cancer patients, IFN- $\gamma$ was found to activate TAM tumoricidal activity, inducing a phenotype switch with unequivocal evidence of clinical responses (133). In a model of pancreatic ductal adenocarcinoma, CD40 agonist antibodies promoted a remarkable antitumor effect and induced high expression of M1 markers (MHC class II and CD86) in macrophages (132).

Modulation of macrophage function is an off-target effect for a number of diverse therapeutic agents. PPAR $\gamma$ agonists (thiazolidinediones) have long been used in the treatment of diabetes. The evidence linking PPAR $\gamma$ to M2 polarization and hence to the homeostatic role of ATMs sheds fresh new light on their mode of action. Preclinical evidence suggests that PPAR $\gamma$ promotes
M2-like polarization and homeostatic metabolic function in ATMs and that alteration of this function is a key pathogenic feature in diabetes (134-136). Other therapeutic strategies that have been reported to affect macrophage polarization include zoledronic acid (an agent used for preventing recurrence of breast cancer bone metastasis), statins (137), trabectedin (138) and TLR ligands (e.g., imiquimod and CpG) (see also Supplemental References).

\section{Concluding remarks}

Progress has been made in defining the molecular networks underlying polarized activation of macrophages. Molecular determinants of M1 versus M2 polarization include members of the PPAR, KLF, IRF, STAT, NF- $\mathrm{B}$, and HIF families. Regulation of skewing also involves epigenetic modifications with involvement of histone methylation and acetylation. miRNAs have emerged as regulators of phagocyte activation and function $(34,139)$, but their role in macrophage polarization needs to be further defined.

Functional polarization has now been observed in vivo under physiological and pathological conditions. The former include embryogenesis and pregnancy and normal maintenance of selected tissues, such as testis and adipose. The latter include chronic inflammation and tissue repair, metabolic and vascular disorders, infection, and cancer. There is now evidence that macrophages are a key component of all of these processes (Figure 2).

Under condition such as parasite infections, allergy, and many cancers, the functional phenotypes of macrophages in vivo mirror those of canonical M1-M2 or M2-like polarized states as defined in vitro. However, in a number of pathological conditions, such as neurodegenerative disorders (140), this is not the case, and macrophage populations express mixed or unique phenotypes. The contribution of coexisting cells with different phenotypes, the impact of dynamic changes during disease evolution, and the molecular networks orchestrating these mixed phenotypes need to be carefully dissected on a case-by-case basis. Indeed, this macrophage plasticity calls for in-depth analysis in different pathological conditions.

Therapeutic macrophage targeting is in its infancy. Selected clinically approved therapeutic strategies, such as use of PPAR $\gamma$ inhibitors, statins, zolendronic acid, and preventive activities such as weight loss may have an impact on the functional status of macrophages; however, the extent to which their effect on macrophages explains their clinical efficacy remains to be defined. The identification of mechanisms and molecules associated with macrophage plasticity and polarized activation provides a basis for macrophage-centered diagnostic and therapeutic strategies.

\section{Acknowledgments}

This work was supported by Associazione Italiana Ricerca sul Cancro (AIRC), Italy; Fondazione Cariplo, Italy; Ministero Università Ricerca (MIUR) e Salute; and by the European Commission (ERC Project No. 233417 HIIS to AM).

Address correspondence to: Antonio Sica, IRCCS Istituto Clinico Humanitas, via Manzoni 113, 20089 Rozzano, Milan, Italy. Phone: 39.02.8224.5111; Fax: 39.02.8224.5101; E-mail: antonio.sica@ humanitasresearch.it.
1. Gordon S, Martinez FO. Alternative activation of macrophages: mechanism and functions. Immunity. 2010;32(5):593-604.

2. Biswas SK, Mantovani A. Macrophage plasticity and interaction with lymphocyte subsets: cancer as a paradigm. Nat Immunol. 2010;11(10):889-896.

3. Sica A, Bronte V. Altered macrophage differentiation and immune dysfunction in tumor development.
J Clin Invest. 2007;117(5):1155-1166

4. Mantovani A, Sozzani S, Locati M, Allavena P, Sica A. Macrophage polarization: tumor-associated macrophages as a paradigm for polarized 
M2 mononuclear phagocytes. Trends Immunol. 2002;23(11):549-555

5. Biswas SK, Mantovani A. Macrophage plasticity and interaction with lymphocyte subsets: cancer as a paradigm. Nat Immunol. 2010;11(10):889-896.

6. Mosser DM, Edwards JP. Exploring the full spectrum of macrophage activation. Nat Rev Immunol. 2008;8(12):958-969.

7. Saccani A, et al. p50 nuclear factor-kappaB overexpression in tumor-associated macrophages inhibits M1 inflammatory responses and antitumor resistance. Cancer Res. 2006;66(23):11432-11440.

8. Guiducci C, Vicari AP, Sangaletti S, Trinchieri G, Colombo MP. Redirecting in vivo elicited tumor infiltrating macrophages and dendritic cells towards tumor rejection. Cancer Res. 2005;65(8):3437-3446

9. Martinez FO, Helming L, Gordon S. Alternative activation of macrophages: an immunologic functional perspective. Annu Rev Immunol. 2009;27:451-483.

10. O'Shea JJ, Paul WE. Mechanisms underlying lineage commitment and plasticity of helper CD 4+ T cells. Science. 2010;327(5969):1098-1102.

11. Murray PJ, Wynn TA. Obstacles and opportunities for understanding macrophage polarization.J Leukoc Biol. 2011;89(4):557-563

12. Krausgruber T, et al. IRF5 promotes inflammatory macrophage polarization and TH1-TH17 responses. Nat Immunol. 2011;12(3):231-238.

13. Junttila IS, et al. Tuning sensitivity to IL-4 and IL-13: differential expression of IL-4Ralpha, IL-13Ralpha1, and gammac regulates relative cytokine sensitivity. J Exp Med. 2008;205(11):2595-2608.

14. Pauleau AL, Rutschman R, Lang R, Pernis A, Watowich SS, Murray PJ. Enhancer-mediated control of macrophage-specific arginase I expression. JImmunol. 2004;172(12):7565-7573

15. Lang R, Patel D, Morris JJ, Rutschman RL, Murray $\mathrm{PJ}$. Shaping gene expression in activated and resting primary macrophages by IL-10.J Immunol. 2002;169(5):2253-2263.

16. Gordon S. Alternative activation of macrophages. Nat Rev Immunol. 2003;3(1):23-35.

17. Whyte CS, et al. Suppressor of cytokine signaling (SOCS) 1 is a key determinant of differential macrophage activation and function. J Leukoc Biol. 2011;90(5):845-854.

18. Liu Y, et al. Unique expression of suppressor of cytokine signaling 3 is essential for classical macrophage activation in rodents in vitro and in vivo. J Immunol. 2008;180(9):6270-6278.

19. Odegaard JI, et al. Macrophage-specific PPARgamma controls alternative activation and improves insulin resistance. Nature. 2007;447(7148):1116-1120.

20. Odegaard JI, et al. Alternative M2 activation of Kupffer cells by PPARdelta ameliorates obesity-induced insulin resistance. Cell Metab. 2008;7(6):496-507.

21. Kang K, et al. Adipocyte-derived Th2 cytokines and myeloid PPARdelta regulate macrophage polarization and insulin sensitivity. Cell Metab. 2008;7(6):485-495.

22. Szanto A, et al. STAT6 transcription factor is a facilitator of the nuclear receptor PPARgammaregulated gene expression in macrophages and dendritic cells. Immunity. 2010;33(5):699-712.

23. Cao Z, Sun X, Icli B, Wara AK, Feinberg MW. Role of Kruppel-like factors in leukocyte development, function, and disease. Blood. 2010; 116(22):4404-4414

24. Liao X, et al. Kruppel-like factor 4 regulates macrophage polarization. J Clin Invest. 2011; 121(7):2736-2749.

25. Mahabeleshwar GH, et al. The myeloid transcription factor KLF2 regulates the host response to polymicrobial infection and endotoxic shock. Immunity. 2011;34(5):715-728.

26. Pello OM, et al. Role of c-Myc in alternative activation of human macrophages and tumor-associated macrophage biology. Blood. 2012;119(2):411-421.

27. Bonizzi G, Karin M. The two NF-kappaB activation pathways and their role in innate and adaptive immunity. Trends Immunol. 2004;25(6):280-288.

28. Lawrence T, Gilroy DW. Chronic inflammation: a failure of resolution? Int J Exp Pathol. 2007;88(2):85-94.

29. Hagemann T, et al. "Re-educating" tumor-associated macrophages by targeting NF-kappaB. J Exp Med. 2008;205(6):1261-1268.

30. Porta C, et al. Tolerance and M2 (alternative) macrophage polarization are related processes orchestrated by 550 nuclear factor kappaB. Proc Natl Acad Sci U S A. 2009;106(35):14978-14983.

31. Takeda N, et al. Differential activation and antagonistic function of HIF-\{alpha\} isoforms in macrophages are essential for NO homeostasis. Genes Dev. 2010;24(5):491-501.

32. Satoh T, et al. The Jmjd3-Irf4 axis regulates M2 macrophage polarization and host responses against helminth infection. Nat Immunol. 2010; 11(10):936-944.

33. Ishii $\mathrm{M}$, et al. Epigenetic regulation of the alternatively activated macrophage phenotype. Blood. 2009;114(15):3244-3254

34. Quinn SR, O'Neill LA. A trio of microRNAs that control Toll-like receptor signalling. Int Immunol. 2011;23(7):421-425.

35. Martinez-Nunez RT, Louafi F, Sanchez-Elsner T. The interleukin 13 (IL-13) pathway in human macrophages is modulated by microRNA- 155 via direct targeting of interleukin 13 receptor alpha 1 (IL13Ralpha1).J Biol Chem. 2011;286(3):1786-1794.

36. Lucas $T$, et al. Differential roles of macrophages in diverse phases of skin repair. J Immunol. 2010; 184(7):3964-3977.

37. Serhan $\mathrm{CN}$, et al. Novel proresolving aspirin-triggered DHA pathway. Chem Biol. 2011;18(8):976-987.

38. Mares CA, et al. Defect in efferocytosis leads to alternative activation of macrophages in Francisella infections. Immunol Cell Biol. 2011;89(2):167-172.

39. Al-Mulla F, Leibovich SJ, Francis IM, Bitar MS. Impaired TGF-beta signaling and a defect in resolution of inflammation contribute to delayed wound healing in a female rat model of type 2 diabetes. Mol Biosyst. 2011;7(11):3006-3020.

40. Bystrom J, et al. Resolution-phase macrophages possess a unique inflammatory phenotype that is controlled by cAMP. Blood. 2008;112(10):4117-4127.

41. Sindrilaru A, et al. An unrestrained proinflammatory M1 macrophage population induced by iron impairs wound healing in humans and mice. J Clin Invest. 2011;121(3):985-997.

42. Cairo G, Recalcati S, Mantovani A, Locati M. Iron trafficking and metabolism in macrophages: contribution to the polarized phenotype. Trends Immunol. 2011;32(6):241-247.

43. Kobayashi M, et al. M2b monocytes predominated in peripheral blood of severely burned patients. J Immunol. 2010;185(12):7174-7179.

44. Swirski FK, et al. Identification of splenic reservoir monocytes and their deployment to inflammatory sites. Science. 2009;325(5940):612-616.

45. Takeda Y, et al. Macrophage skewing by Phd 2 haplodeficiency prevents ischaemia by inducing arteriogenesis. Nature. 2011;479(7371):122-126.

46. London A, et al. Neuroprotection and progenitor cell renewal in the injured adult murine retina requires healing monocyte-derived macrophages. J Exp Med. 2011;208(1):23-39.

47. Paulson JC. Innate immune response triggers lupus-like autoimmune disease. Cell. 2007; 130(4):589-591.

48. Zhang W, Xu W, Xiong S. Blockade of Notch1 signaling alleviates murine lupus via blunting macrophage activation and $\mathrm{M} 2 \mathrm{~b}$ polarization. J Immunol. 2010;184(11):6465-6478.

49. Zhang W, Xu W, Xiong S. Macrophage differentiation and polarization via phosphatidylinositol
3-kinase/Akt-ERK signaling pathway conferred by serum amyloid P component. J Immunol. 2011; 187(4):1764-1777.

50. Vandooren B, et al. Absence of a classically activated macrophage cytokine signature in peripheral spondylarthritis, including psoriatic arthritis. Arthritis Rheum. 2009;60(4):966-975.

51. Haringman JJ, et al. Synovial tissue macrophages: a sensitive biomarker for response to treatment in patients with rheumatoid arthritis. Ann Rheum Dis. 2005;64(6):834-838.

52. Cuzzocrea S, et al. Reduction in the evolution of murine type II collagen-induced arthritis by treatment with rosiglitazone, a ligand of the peroxisome proliferator-activated receptor gamma. Arthritis Rheum. 2003;48(12):3544-3556.

53. Shirinsky IV, Shirinsky VS. Targeting nuclear hormone receptors: PPARalpha agonists as potential disease-modifying drugs for rheumatoid arthritis. Int J Rheumatol. 2011;2011:937843.

54. Waddell A, et al. Colonic eosinophilic inflammation in experimental colitis is mediated by Ly6C(high) CCR2 (+) inflammatory monocyte/macrophagederived CCL11. J Immunol. 2011;186(10):5993-6003.

55. Shaughnessy LM, Swanson JA. The role of the activated macrophage in clearing Listeria monocytogenes infection. Front Biosci. 2010;12:2683-2692.

56. Jouanguy E, Doffinger R, Dupuis S, Pallier A, Altare F, Casanova JL. IL-12 and IFN-gamma in host defense against mycobacteria and salmonella in mice and men. Curr Opin Immunol. 1999;11(3):346-351.

57. Chacon-Salinas R, et al. Differential pattern of cytokine expression by macrophages infected in vitro with different Mycobacterium tuberculosis genotypes. Clin Exp Immunol. 2005;140(3):443-449.

58. Kiszewski AE, et al. The local immune response in ulcerative lesions of Buruli disease. Clin Exp Immunol. 2006;143(3):445-451.

59. Murphy JT, et al. Gene expression profiling of monocyte-derived macrophages following infection with Mycobacterium avium subspecies avium and Mycobacterium avium subspecies paratuberculosis. Physiol Genomics. 2006;28(1):67-75.

60. Cavaillon JM, Adib-Conquy M. Bench-to-bedside review: endotoxin tolerance as a model of leukocyte reprogramming in sepsis. Crit Care. 2006; 10(5):233.

61. Noel W, Raes G, Hassanzadeh Ghassabeh G, De Baetselier P, Beschin A. Alternatively activated macrophages during parasite infections. Trends Parasitol. 2004;20(3):126-133.

62. Benoit M, Desnues B, Mege JL. Macrophage polarization in bacterial infections. J Immunol. 2008; 181(6):3733-3739.

63. Pathak SK, et al. Direct extracellular interaction between the early secreted antigen ESAT- 6 of Mycobacterium tuberculosis and TLR2 inhibits TLR signaling in macrophages. Nat Immunol. 2007; 8(6):610-618

64. Benoit M, Barbarat B, Bernard A, Olive D, Mege JL. Coxiella burnetii, the agent of $\mathrm{Q}$ fever, stimulates an atypical M2 activation program in human macrophages. Eur J Immunol. 2008;38(4):1065-1070.

65 . Helming L, Gordon S. Molecular mediators of macrophage fusion. Trends Cell Biol. 2009;19(10):514-522.

66 . Van den Bossche J, et al. Alternatively activated macrophages engage in homotypic and heterotypic interactions through IL-4 and polyamine-induced E-cadherin/catenin complexes. Blood. 2009; 114(21):4664-4674

67. Babu S, Blauvelt CP, Kumaraswami V, Nutman TB. Chemokine receptors of T cells and of B cells in lymphatic filarial infection: a role for CCR9 in pathogenesis. J Infect Dis. 2005;191(6):1018-1026.

68 . Brys L, et al. Reactive oxygen species and 12/15lipoxygenase contribute to the antiproliferative capacity of alternatively activated myeloid cells elicited during helminth infection. J Immunol. 2005; 
174(10):6095-6104.

69. Pearce EJ, MacDonald AS. The immunobiology of schistosomiasis. Nat Rev Immunol. 2002; 2(7):499-511.

70. Herbert DR, et al. Alternative macrophage activation is essential for survival during schistosomiasis and downmodulates $\mathrm{T}$ helper 1 responses and immunopathology. Immunity. 2004;20(5):623-635.

71. Shirey KA, et al. Control of RSV-induced lung injury by alternatively activated macrophages is IL-4R alpha-, TLR4-, and IFN-beta-dependent. Mucosal Immunol. 2010;3(3):291-300.

72. Kim HY, DeKruyff RH, Umetsu DT. The many paths to asthma: phenotype shaped by innate and adaptive immunity. Nat Immunol. 2010;11(7):577-584.

73. Melgert BN, ten Hacken NH, Rutgers B, Timens W, Postma DS, Hylkema MN. More alternative activation of macrophages in lungs of asthmatic patients. J Allergy Clin Immunol. 2011;127(3):831-833.

74. Trujillo G, O'Connor EC, Kunkel SL, Hogaboam CM. A novel mechanism for CCR4 in the regulation of macrophage activation in bleomycin-induced pulmonary fibrosis. Am J Pathol. 2008;172(5):1209-1221.

75. Zhu Z, et al. Acidic mammalian chitinase in asthmatic Th2 inflammation and IL-13 pathway activation. Science. 2004;304(5677):1678-1682.

76. Reese TA, et al. Chitin induces accumulation in tissue of innate immune cells associated with allergy. Nature. 2007;447(7140):92-96.

77. Wynn TA.IL-13 effector functions. Annu RevImmunol. 2003:21:425-456.

78. Ather JL, et al. Serum amyloid A activates the NLRP3 inflammasome and promotes Th17 allergic asthma in mice. J Immunol. 2011;187(1):64-73.

79. Nambu A, Nakae S. IL-1 and Allergy. Allergol Int. 2010; 59(2):125-135

80. Tsutsui H, Yoshimoto T, Hayashi N, Mizutani H, Nakanishi K. Induction of allergic inflammation by interleukin-18 in experimental animal models. Immunol Rev. 2004;202:115-138.

81. Moreira AP, Hogaboam CM. Macrophages in allergic asthma: fine-tuning their pro- and anti-inflammatory actions for disease resolution.J Interferon Cytokine Res. 2011;31(6):485-491.

82. Mantovani A, Allavena P, Sica A, Balkwill F. Cancer-related inflammation. Nature. 2008; 454(7203):436-444.

83. Qian BZ, et al. CCL2 recruits inflammatory monocytes to facilitate breast-tumour metastasis. Nature. 2011;475(7355):222-225.

84. Zaynagetdinov R, et al. A critical role for macrophages in promotion of urethane-induced lung carcinogenesis. J Immunol. 2011;187(11):5703-5711.

85 . Kang TW, et al. Senescence surveillance of pre-malignant hepatocytes limits liver cancer development. Nature. 2011;479(7374):547-551.

86. Schreiber RD, Old LJ, Smyth MJ. Cancer immunoediting: integrating immunity's roles in cancer suppression and promotion. Science. 2011; 331(6024):1565-1570.

87. Steidl C, et al. Tumor-associated macrophages and survival in classic Hodgkin's lymphoma. NEnglJMed. 2010;362(10):875-885.

88. Chen J, et al. CCL18 from tumor-associated macrophages promotes breast cancer metastasis via PITPNM3. Cancer Cell. 2011;19(4):541-555.

89. Movahedi K, et al. Different tumor microenvironments contain functionally distinct subsets of macrophages derived from Ly6C(high) monocytes. Cancer Res. 2010;70(14):5728-5739.

90. Schioppa T, et al. B regulatory cells and the tumor-promoting actions of TNF-alpha during squamous carcinogenesis. Proc Natl Acad Sci U S A. 2011;108(26):10662-10667.

91. Andreu P, et al. FcRgamma activation regulates inflammation-associated squamous carcinogenesis. Cancer Cell. 2010;17(2):121-134.

92. DeNardo DG, et al. CD4(+) T cells regulate pul- monary metastasis of mammary carcinomas by enhancing protumor properties of macrophages. Cancer Cell. 2009;16(2):91-102.

93. Pedroza-Gonzalez A, et al. Thymic stromal lymphopoietin fosters human breast tumor growth by promoting type 2 inflammation. J Exp Med. 2011;208(3):479-490.

94. Erez N, Truitt M, Olson P, Arron ST, Hanahan D. Cancer-associated fibroblasts are activated in incipient neoplasia to orchestrate tumor-promoting inflammation in an NF-kappaB-dependent manner. Cancer Cell. 2010;17(2):135-147.

95. Erler JT, et al. Hypoxia-induced lysyl oxidase is a critical mediator of bone marrow cell recruitment to form the premetastatic niche. Cancer Cell. 2009; 15(1):35-44.

96. Kim S, et al. Carcinoma-produced factors activate myeloid cells through TLR2 to stimulate metastasis. Nature. 2009;457(7225):102-106.

97. Roca H, Varsos ZS, Sud S, Craig MJ, Ying C, Pienta KJ. CCL2 and interleukin- 6 promote survival of human $\mathrm{CD} 11 \mathrm{~b}+$ peripheral blood mononuclear cells and induce M2-type macrophage polarization. J Biol Chem. 2009;284(49):34342-34354.

98. Jinushi M, et al. Tumor-associated macrophages regulate tumorigenicity and anticancer drug responses of cancer stem/initiating cells. Proc Natl Acad Sci U S A. 2011;108(30):12425-12430.

99. Hotamisligil GS. Inflammation and metabolic disorders. Nature. 2006;444(7121):860-867.

100. Olefsky JM, Glass CK. Macrophages, inflammation, and insulin resistance. Annu Rev Physiol. 2010;72:219-246

101. Weisberg SP, McCann D, Desai M, Rosenbaum M, Leibel RL, Ferrante AW Jr. Obesity is associated with macrophage accumulation in adipose tissue. J Clin Invest. 2003;112(12):1796-1808.

102. Xu H, et al. Chronic inflammation in fat plays a crucial role in the development of obesity-related insulin resistance. J Clin Invest. 2003;112(12):1821-1830.

103. Weisberg SP, et al. CCR2 modulates inflammatory and metabolic effects of high-fat feeding. J Clin Invest. 2006;116(1):115-124.

104. Kanda H, et al. MCP-1 contributes to macrophage infiltration into adipose tissue, insulin resistance, and hepatic steatosis in obesity. J Clin Invest. 2006;116(6):1494-1505.

105. Kurokawa J, et al. Apoptosis inhibitor of macrophage (AIM) is required for obesity-associated recruitment of inflammatory macrophages into adipose tissue. Proc Natl Acad Sci U S A. 2011; 108(29):12072-12077.

106. Hevener AL, et al. Macrophage PPAR gamma is required for normal skeletal muscle and hepatic insulin sensitivity and full antidiabetic effects of thiazolidinediones. JClin Invest. 2007;117(6):1658-1669.

107. Lumeng CN, Bodzin JL, Saltiel AR. Obesity induces a phenotypic switch in adipose tissue macrophage polarization. J Clin Invest. 2007;117(1):175-184.

108. Shaul ME, Bennett G, Strissel KJ, Greenberg AS, Obin MS. Dynamic, M2-like remodeling phenotypes of $\mathrm{CD} 11 \mathrm{c}^{+}$adipose tissue macrophages during high-fat diet--induced obesity in mice. Diabetes. 2010;59(5):1171-1181.

109. Kosteli A, et al. Weight loss and lipolysis promote a dynamic immune response in murine adipose tissue. J Clin Invest. 2010;120(10):3466-3479.

110.Odegaard JI, Chawla A. Alternative macrophage activation and metabolism. Annu Rev Pathol. 2011; 6:275-297.

111. Hu X, et al. Integrated regulation of Toll-like receptor responses by Notch and interferon-gamma pathways. Immunity. 2008;29(5):691-703.

112. Marathe $\mathrm{C}$, et al. Preserved glucose tolerance in high-fat-fed C57BL/ 6 mice transplanted with PPARgamma $^{-/}$, PPARdelta- ${ }^{-/}$, PPARgammadel$\mathrm{ta}^{-/}$, or LXRalphabeta-/- bone marrow. J Lipid Res. 2009;50(2):214-224.
113.Nguyen KD, et al. Alternatively activated macrophages produce catecholamines to sustain adaptive thermogenesis. Nature. 2011;480(7375):104-108.

114. Wang JM, Sherry B, Fivash MJ, Kelvin DJ, Oppenheim JJ. Human recombinant macrophage inflammatory protein-1 alpha and -beta and monocyte chemotactic and activating factor utilize common and unique receptors on human monocytes. J Immunol. 1993;150(7):3022-3029.

115. Barleon B, Sozzani S, Zhou D, Weich HA, Mantovani A, Marme D. Migration of human monocytes in response to vascular endothelial growth factor (VEGF) is mediated via the VEGF receptor flt-1. Blood. 1996;87(8):3336-3343.

116. Ohno $\mathrm{H}$, et al. A c-fms tyrosine kinase inhibitor, Ki20227, suppresses osteoclast differentiation and osteolytic bone destruction in a bone metastasis model. Mol Cancer Ther. 2006;5(11):2634-2643.

117. Manthey CL, et al. JNJ-28312141, a novel orally active colony-stimulating factor-1 receptor/FMS-related receptor tyrosine kinase-3 receptor tyrosine kinase inhibitor with potential utility in solid tumors, bone metastases, and acute myeloid leukemia. Mol Cancer Ther. 2009;8(11):3151-3161.

118. Martinez FO, Gordon S, Locati M, Mantovani A. Transcriptional profiling of the human monocyteto-macrophage differentiation and polarization: new molecules and patterns of gene expression. JImmunol. 2006;177(10):7303-7311.

119.Zhang J, Lu Y, Pienta KJ. Multiple roles of chemokine (C-C motif) ligand 2 in promoting prostate cancer growth. J Natl Cancer Inst. 2010;102(8):522-528.

120.Loberg RD, et al. Targeting CCL2 with systemic delivery of neutralizing antibodies induces prostate cancer tumor regression in vivo. Cancer Res. 2007; 67(19):9417-9424.

121. Gazzaniga S, et al. Targeting tumor-associated macrophages and inhibition of MCP-1 reduce angiogenesis and tumor growth in a human melanoma xenograft. J Invest Dermatol. 2007;127(8):2031-2041.

122.Aharinejad $S$, et al. Colony-stimulating factor-1 antisense treatment suppresses growth of human tumor xenografts in mice. Cancer Res. 2002; 62(18):5317-5324.

123. Aharinejad S, et al. Colony-stimulating factor-1 blockade by antisense oligonucleotides and small interfering RNAs suppresses growth of human mammary tumor xenografts in mice. Cancer Res. 2004; 64(15):5378-5384.

124. Paulus P, Stanley ER, Schafer R, Abraham D, Aharinejad S. Colony-stimulating factor- 1 antibody reverses chemoresistance in human MCF-7 breast cancer xenografts. Cancer Res. 2006;66(8):4349-4356.

125.Giraudo E, Inoue M, Hanahan D. An aminobisphosphonate targets MMP-9-expressing macrophages and angiogenesis to impair cervical carcinogenesis. J Clin Invest. 2004;114(5):623-633.

126.Ruffell B, Au A, Rugo HS, Esserman LJ, Hwang ES, Coussens LM. Leukocyte composition of human breast cancer [published online ahead of print August 8, 2011]. Proc Natl Acad Sci U S A. doi:10.1073/pnas.1104303108.

127. Davies LC, Rosas M, Smith PJ, Fraser DJ, Jones SA, Taylor PR. A quantifiable proliferative burst of tissue macrophages restores homeostatic macrophage populations after acute inflammation. Eur J Immunol. 2011;41(8):2155-2164.

128.Jenkins SJ, et al. Local macrophage proliferation, rather than recruitment from the blood, is a signature of TH2 inflammation. Science. 2011; 332(6035):1284-1288.

129. Mantovani A, Sica A. Macrophages, innate immunity and cancer: balance, tolerance, and diversity. Curr Opin Immunol. 2010;22(2):231-237.

130. Sica A, et al. Autocrine production of IL-10 mediates defective IL-12 production and NF-kappa $\mathrm{B}$ activation in tumor-associated macrophages. J Immunol. 2000;164(2):762-767. 
131. Duluc D, et al. Interferon-gamma reverses the immunosuppressive and protumoral properties and prevents the generation of human tumor-associated macrophages. Int J Cancer. 2009;125(2):367-373

132. Beatty GL, et al. CD40 agonists alter tumor stroma and show efficacy against pancreatic carcinoma in mice and humans. Science. 2011; 331(6024):1612-1616.

133.Allavena $\mathrm{P}$, et al. Intraperitoneal recombinant gamma-interferon in patients with recurrent ascitic ovarian carcinoma: modulation of cytotoxicity and cytokine production in tumorassociated effectors and of major histocompatibility antigen expression on tumor cells. Cancer Res. 1990;50(22):7318-7323.

134.Lu M, et al. Brain PPAR-gamma promotes obesity and is required for the insulin-sensitizing effect of thiazolidinediones. Nat Med. 2011;17(5):618-622.

135.Stienstra R, Duval C, Keshtkar S, van der Laak J,
Kersten S, Muller M. Peroxisome proliferatoractivated receptor gamma activation promotes infiltration of alternatively activated macrophages into adipose tissue. J Biol Chem. 2008; 283(33):22620-22627.

136. Charo IF. Macrophage polarization and insulin resistance: PPARgamma in control. Cell Metab. 2007;6(2):96-98.

137. Fujita E, et al. Statin attenuates experimental anti-glomerular basement membrane glomerulonephritis together with the augmentation of alternatively activated macrophages. Am J Pathol. 2010; 177(3):1143-1154.

138. Germano G, et al. Antitumor and anti-inflammatory effects of trabectedin on human myxoid liposarcoma cells. Cancer Res. 2010;70(6):2235-2244.

139. Bazzoni F, et al. Induction and regulatory function of miR-9 in human monocytes and neutrophils exposed to proinflammatory signals. Proc Natl Acad
Sci US A. 2010;106(13):5282-5287.

140. Robert S, et al. Severe diabetes, age-dependent loss of adipose tissue, and mild growth deficiency in mice lacking $\mathrm{Akt} 2 / \mathrm{PKB} \beta . J$ Clin Invest. 2003;112(2):197-208

141.Lai J-J, et al. Monocyte/macrophage androgen receptor suppresses cutaneous wound healing in mice by enhancing local TNF- $\alpha$ expression. J Clin Invest. 2009;119(12):3739-3751.

142. Yu M, et al. Identification of an IFN- $\gamma /$ mast cell axis in a mouse model of chronic asthma. J Clin Invest. 2011;121(8):3133-3143

143. Onoyama I, et al. Fbxw7 regulates lipid metabolism and cell fate decisions in the mouse liver. JClin Invest. 2011;121(1):342-354

144.Prados-Rosales R, et al. Mycobacteria release active membrane vesicles that modulate immune responses in a TLR2-dependent manner in mice. JClin Invest. 2011;121(4):1471-1483. 